July 23, 2016

Prof. Reuben H. Simoyi, Department of Chemistry, Portland State University, Portland, OR 97207-0751, USA.

\title{
Electrochemistry-Coupled to Mass Spectrometry in Simulation of Metabolic Oxidation of Methimazole: Identification and characterization of metabolites
}

by

\footnotetext{
Kudzanai Chipiso ${ }^{+}$and Reuben H. Simoyi ${ }^{*+\neq}$

'Department of Chemistry, Portland State University, Portland, OR 97207-0751, USA.

‡School of Chemistry and Physics, University of KwaZulu-Natal, Westville Campus, Durban 4014. South

Africa.
} 


\begin{abstract}
Methimazole (MMI), an antithyroid drug, is associated with idiosyncratic toxicity. Reactive metabolites resulting from bioactivation of the drug have been implicated in these adverse drug reactions. Mimicry of enzymatic oxidation of MMI was carried out by electrochemically oxidizing MMI using a coulometric flow-through cell equipped with a porous graphite working electrode. The cell was coupled on-line to electrospray ionization mass spectrometry (EC/ESI-MS. ESI spectra were acquired in both negative and positive modes. In acidic medium, ESI spectral analysis showed that the dimer was the main product, while in neutral and basic media, methimazole sulfenic acid, methimazole sulfinic acid and methimazole sulfonic acid were observed as the major electrochemical oxidation products. Oxidation of $\mathrm{MMI}$ and subsequent trapping with nucleophiles resulted in formation of adducts with $\mathrm{N}$-acetylcysteine. Some of the electrochemically generated species observed in these experiments were similar to metabolites that have been observed from in vitro and in vivo studies. Trapping studies also showed that bioactivation of MMI proceeds predominantly through the S-oxide and not through formation of thyil radicals. These results show that electrochemistry coupled to mass spectrometry can be used in mimicry of oxidative metabolism and subsequent high throughput screening of metabolites.
\end{abstract}

Key words: electrochemistry, oxidation, mass spectrometry, reactive metabolites 
Graphical Abstract.

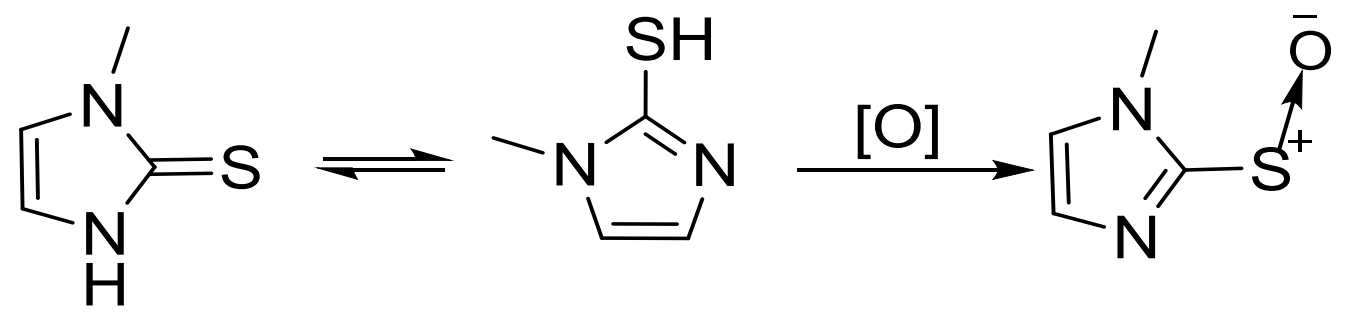




\section{Introduction}

The conventional method of studying oxidative drug metabolism during preclinical experiments is through animal models (in vivo) or perfused organs in vitro. ${ }^{1}$ However, the use of animals in experiments involving scientific research and biological testing has raised concerns over the years among animal advocates. ${ }^{2}$ In view of this as well as the large number of drug candidates emerging and those existing, there has been a renewed interest in development of complementary tools for mimicry of oxidative metabolism. Although it remains a challenge to extrapolate data generated from such systems to actual In vivo systems, these biomimetic tools offer some advantages that are not inherent in conventional methods. Electrochemistry coupled on-line to liquid chromatograph/mass spectrometer (EC/LC/MS or $\mathrm{EC} / \mathrm{MS}$ ) has the potential to mimic redox metabolism. ${ }^{3-6}$ Members of the cytochromes $\mathrm{P} 450$ class (CYP450) of enzymes are responsible for the majority of phase I biotransformations leading to reactive electrophilic intermediates. ${ }^{7}$ The chemical reactivity of electrophilic metabolites usually prevents their detection in vivo since, by definition, they are short-lived and likely to undergo one or more structural modifications to form more stable final products. Jurva and co-workers investigated the extent to which this technique could be used to mimic cytochrome P450 catalyzed reactions by comparing metabolites generated from EC/LC/MS to those that were generated from the P450 system. Their results showed that reactions such as $\mathrm{N}$-dealkylation, S-oxidation, $\mathrm{P}$-oxidation, alcohol oxidation and dehydrogenation that proceed via a mechanism initiated by one-electron oxidation or hydrogen abstraction are amenable to electrochemical oxidation. ${ }^{8}$

There are a number of therapeutic drugs based on simple sulfur chemistry. Our research in the past has focused on investigating the mechanism of S-oxidation of organosulfur compounds. ${ }^{9,10}$ Extensive studies from our laboratory have shown that nearly every organic sulfur compound presents a unique reactivity and no generic oxidation pathway can be easily derived. Sulfur atom has been implicated as a site of 
bioactivation, resulting in formation of reactive and potentially toxic metabolites from bioactivation of sulfur containing drugs. ${ }^{11}$ Many of the adverse reactions produced by penicillamine and other compounds with an active sulfhydryl group form a distinctive pattern when viewed as a class. Alterations in taste perception, mucocutaneous lesions, proteinuria due to immune-complex membranous glomerulopathy, and pemphigus are adverse reactions that have been encountered with all of these compounds. Classic examples include the thiol, captopril, a still-used antihypertensive drug which comes with a black box warning and is associated with hepatotoxicity. ${ }^{12-14}$ It can only be used in low doses and after careful selection of patients to avoid idiosyncratic drug reactions and thus cannot be used for patients with severe hypertension. ${ }^{13}$ Troglitazone, a thiazolidinedione, developed for diabetes mellitus type 2 , had to be withdrawn from the market because it caused severe liver injury. Thiazoles such as Sudoxicam were withdrawn in Phase III trials when it was already apparent that the drug was rife with IDR's while a closely-related drug, Meloxicam, has not been associated with any IDR's.

Methimazole, an antithyroid drug used in the treatment of hyperthyroidism, has been associated with idiosyncratic toxicity, characterized by skin reactions, leucopenia, agranulocytosis, aplastic anemia, hepatitis and cholestasis. ${ }^{15,16}$ The relationship between idiosyncratic adverse reactions and reactive metabolites is not well established. There is circumstantial evidence, however, that reactive metabolites are involved in the onset of idiosyncratic adverse reactions. ${ }^{17,18}$ We set out to investigate formation of any unexpected or reactive intermediates from MMI. The metabolites are electrophilic in nature, are reactive, and have the capacity to bind to nucleophilic cellular macromolecules which can then elicit an immune response. EC/LC/MS method offers the advantage of generating and isolating reactive intermediates in cellular matrix free environments, since matrix components will immediately bind to the reactive metabolites resulting in evasion of detection. ${ }^{19-21} \mathrm{~A}$ range of metabolites have been suggested during oxidation of MMI. MMI metabolism is thought to occur through a P450-mediated process, resulting in ring scission, with further S-oxidation mediated through FMO to produce the 
tandem of sulfenic and sulfinic acids. ${ }^{22}$ However, some sources reported that FMO sequentially monooxygenates intact methimazole to produce unstable methimazole sulfenic and sulfinic acids without ring scission. ${ }^{23}$

The objective of this study was to use electrochemistry and mass-spectrometry to mimic oxidative metabolism in order to generate and characterize intermediates and products using electrospray ionization. Various electrochemical methods using modified electrodes to enhance catalytic oxidation of $\mathrm{MMI}$ have been developed and used for the determination of $\mathrm{MMI}$ at sub-micromolar detection limits using cyclic voltammetry. ${ }^{24-26}$ While most of these studies were focusing on detection and determination of MMI using electrochemical techniques, our study is designed to explore metabolic fate of MMI. The

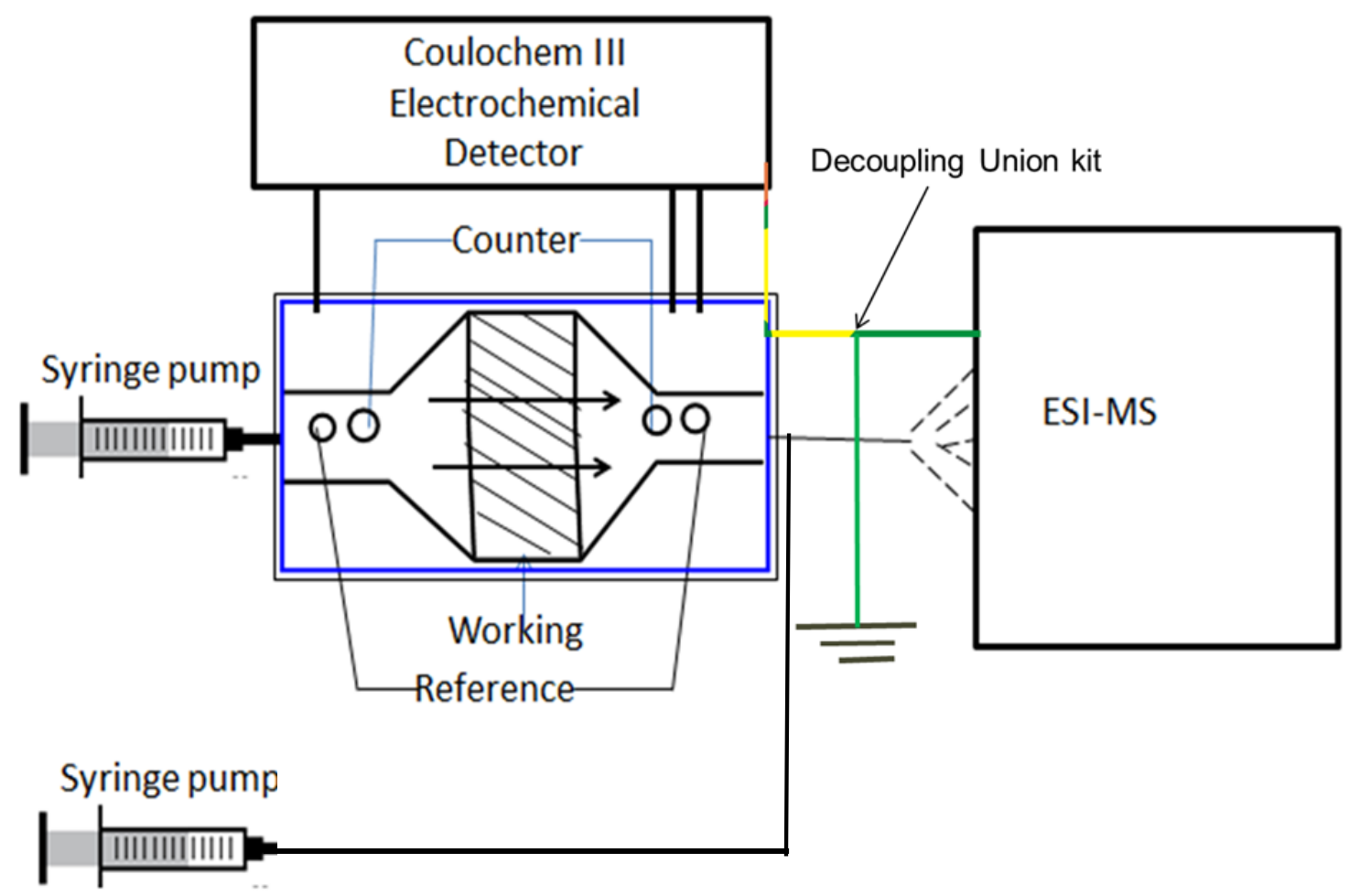

Figure 1.Schematic of EC/ESI-MS system. Coulochem III Electrochemical Detector controls potential of the flow through cell. Samples were infused at $10 \mu \mathrm{L} / \mathrm{min}$. 
electrochemical oxidation of thiol compounds such $\mathrm{MMI}$ is complicated by large anodic over potential and poor voltammetric signals. In the coulometric cell used in this study, the eluent flows through the electrodes rather than by the electrodes as in convectional cells. This maximizes the contact of the electro-active compound in solution with the electrode surface, ensuring that diffusion and convection controlled process do not limit the electrochemical oxidation of the compound. ${ }^{27}$

\section{Experimental Section.}

Reagents: Reagent grade methimazole, reduced glutathione, $\mathrm{N}$-acetylcysteine and methoxylamine were purchased from Sigma Aldrich (USA) and were used without further purification. Water solutions for electrochemical oxidation were purified using a Barnstead Sybron Corp. water purification unit capable of producing both distilled and deionized water (Nanopure). ICPMS, was used to evaluate concentrations of metal ions in the reagent water. ICPMS results showed negligible amounts $(<0.1 \mathrm{ppb})$ of copper, iron and silver ions with approximately $1.5 \mathrm{ppb}$ of cadmium and $0.43 \mathrm{ppb}$ in lead as the highest metal ion concentrations. In previous experiments from our lab, no discernible differences in kinetics data had been obtained between experiments run with chelators (EDTA, deferroxamine) and those run without, and so all experiments were carried out without the use of chelators. Solvents used for electrochemical oxidation and mass spectrometry were HPLC grade.

Instrumentation: Electrochemical oxidations were performed using Thermo Scientific Dionex 5150 Synthesis Cell ${ }^{\mathrm{TM}}$ equipped with a flow through graphite working electrode, solid state palladium reference electrode and a Palladium counter electrode (see Figure 1). The cell potential was controlled using Thermo Scientific Dionex Coulochem III electrochemical detector. The cell outlet was interfaced into a mass spectrometer inlet for on-line analysis using PEEK tubing. To prevent electrical damage to 
the detector and cell; as well as shock, the synthesis cell and the detector were decoupled from the high voltage of the mass spectrometer using a high voltage decoupling union kit. Samples were infused through a syringe pump at a flow rate of $10 \mu \mathrm{L} / \mathrm{min}$ for on-line experiments. Mass spectra of the electrochemical oxidation products, were acquired on a high-resolution $(m / \Delta m=30000)$ Thermo Scientific LTQ-Orbitrap Discovery mass spectrometer (San Jose, CA) equipped with an electrospray ionization source. The MS ESI source parameters were set as follows: spray voltage (kV), 2.5 in negative mode and 4.5 in positive mode; spray current $(\mu \mathrm{A}), 1.96$; sheath gas flow rate, 20; auxiliary gas flow rate, 0.01 ; capillary voltage $(\mathrm{V}),-16$; capillary temperature $\left({ }^{\circ} \mathrm{C}\right), 300$; and tube lens $(\mathrm{V}),-115$. Detection was carried out in both the negative ionization mode and positive (-ESI) for $4 \mathrm{~min}$. The detection parameters were set up as follows: Analyzer; FTMS, positive and negative polarity; mass range; normal, resolution; 30 000, scan type; centroid.

\section{On-line EC/ESI-MS electrochemical oxidation of methimazole.}

Experiments were carried out in acidic, neutral and alkaline medium. $100 \mu \mathrm{M} \mathrm{MMI}$ were dissolved in acidic medium which consisted of $20 \%$ methanol with $80 \% 20$ mM formate buffer ( $\mathrm{pH} 2.75$ ). For neutral medium, a combination of $20 \%$ methanol with $80 \% 50 \mathrm{mM}$ phosphate buffer (pH 7.4) was used. Alkaline media utilized a $20 \%$ methanol with $80 \% 20 \mathrm{mM}$ ammonium buffer solution (pH 10.2). A $500 \mu \mathrm{L}$ sample was infused through the electrochemical cell at a flow rate of $10 \mu \mathrm{L} / \mathrm{min}$ before the cell was turned on. 
Table I: List of structures featured in this manuscript

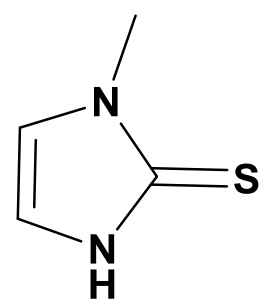

Methimazole

(1-methyl-3H-imidazole-2-thione) (S1)

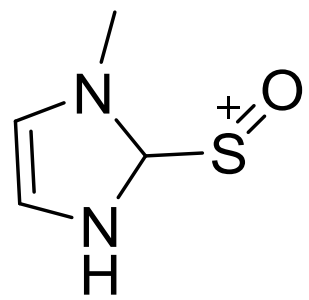

Methimazole S-oxide

(1-methyl-1H-imidazol-2-yl)- $\lambda^{3}$-sulfanone) (M1)<smiles>Cn1ccnc1SSc1nccn1C</smiles>

bis(1-methylimidazol-2-yl)disulfide (M4)<smiles>CSC1NC=CN1C</smiles>

1-methyl-2-(methylthio)-2,3dihydro-1H-imidazole (M7)

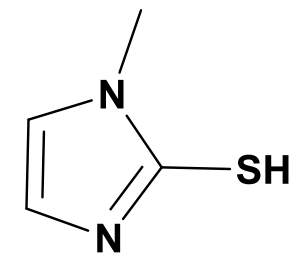

1-methyl-1Himidazole-2-thiol (S2)<smiles>CN1C(=O)CNC1=S</smiles>

3-methyl-2-thioxoimidazolidin-4one (M2)<smiles>CN1C(=O)CNC1=O</smiles>

3-methylimidazolidine-2,4dione ( M3)<smiles>Cn1ccnc1S(=O)[O-]</smiles>

1-methyl-1H-imidazole-2-sulfinate (M5)<smiles>Cn1ccnc1S(=O)(=O)[O-]</smiles>

1-methyl-1H-imidazole2-sulfonate (M6)<smiles>Cn1c(S)nc2c1O2</smiles>

1-methyl-2,3-dihydro-1H- 4-methyl-6-oxa-2,4diazabicyclo[3.1.0]hexa-1(5),2-diene3-thiol (M9) 


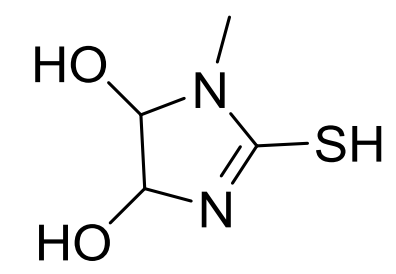

2-mercapto-1-methyl-

1H-imidazole-4,5-diol (M10)

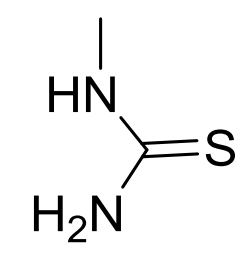

N-methylthiourea (M11)

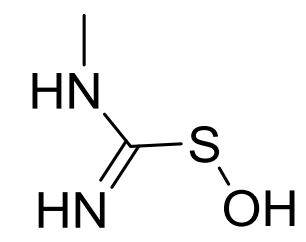

Methylthiourea

sulfenic acid (M12)<smiles>CNC(=N)S(=O)O</smiles>

$\mathrm{N}$-methylthiourea

sulfonic acid (M13)

Table I legend: Those labeled with a prefix of 'S' indicate different forms of the substrate, methimazole, and those with ' $\mathrm{M}$ ' indicate metabolites, either observed in this study or in previous studies.

With the cell turned on, species generated from oxidation in each medium were monitored on the mass spectrum generated on-line. The potential was changed manually on the front panel of control module of ESA Coulochem III Electrochemical detector from $100 \mathrm{mv}$ to $1200 \mathrm{mv}$. Each scan acquisition lasted for four minutes. The optimum potential was determined to be $600 \mathrm{mV}$ vs Pd solid state reference. Higher potentials resulted in a large number of unidentifiable fragments.

\section{Choice of supporting electrolyte and effect of $\mathrm{pH}$}

Larger currents were attained in phosphate and in ammonium buffers. The same species obtained at pH 7.4 in phosphate were also obtained in more alkaline media of $\mathrm{pH}$ 10.2. Phosphate buffer, however, was not used for subsequent experiments. This was because phosphate buffers are non-volatile and can clog the MS inlet capillary, and hence ammonium buffer was chosen as the suitable supporting electrolyte in subsequent experiments. In acidic medium the dimer was the predominant metabolite.

\section{Assessment of stability, reactivity of metabolites and off-line synthesis}


In order to perform off-line synthesis of intermediates, stability of the electrochemically generated was monitored over a period of $30 \mathrm{~h}$. To collect samples off-line, samples were infused at $40 \mu \mathrm{L} / \mathrm{min}$, with potential maintained at $600 \mathrm{mV}$. The samples were then collected in vials with one batch kept at room temperature and the other batch kept at $-20^{\circ} \mathrm{C}$. Samples were analyzed by ESI-MS. Relative abundances of the intermediates at $\mathrm{m} / \mathrm{z} 129$ and 146 decreased significantly after $26 \mathrm{~h}$. for both the samples that were kept frozen at $-20^{\circ} \mathrm{C}$ and those maintained at room temperature. There was a $75 \%$ decrease in abundance of these intermediates over this time duration. In addition, stability of MMI was also monitored and was found to be stable for prolonged periods in solution.

S-oxidation reactions in vitro had revealed that reactive intermediates are produced during oxidation of $\mathrm{MMI}$ and these can be eliminated by addition of nucleophiles such as glutathione to the reaction mixture ${ }^{22}$. In order to check reactivity of the intermediate species, samples were also incubated with a selected set of nucleophiles. Conjugates were observed when the samples were incubated with Nacetylcysteine. Methoxylamine and glutathione, however did not form conjugates with $\mathrm{MMI}$ metabolites. 


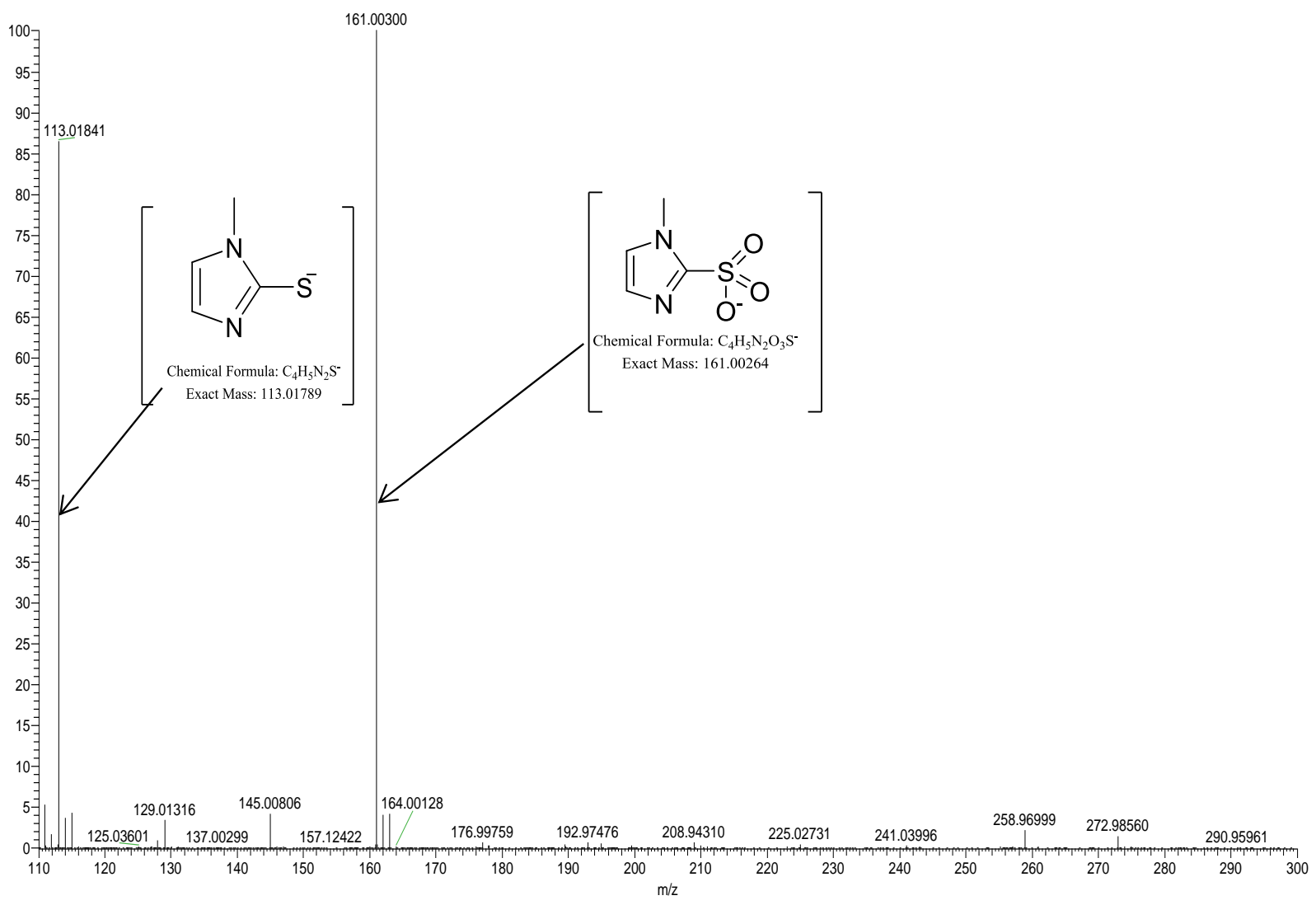

Figure 2. On-line negative mode ESI-MS analysis of methimazole oxidized in ammonium buffer. MMI was infused at $10 \mu \mathrm{L} /$ minute. At this low flow rate the substrate has sufficient residence time in the oxidation cell allowing complete oxidation to sulfonic acid.

\section{Results.}

Technical limitations with respect to the spectrometer cavity could not allow for a faster flow rate than $10 \mu \mathrm{L}$ per minute. Thus the residence time of the substrate in the oxidation chamber was generally long enough for full oxidation of MMI to its full oxidation products of the sulfonic acid at $600 \mathrm{mV}$. The reaction could be slowed down by running the oxidation in basic environments. Figure 2 shows the online negative mode ESI spectrum obtained. This spectrum shows a strong peak of the sulfonic acid at $\mathrm{m} / \mathrm{z}=161.003$ and another for the unoxidized substrate, $\mathrm{MMI}$ at $\mathrm{m} / \mathrm{z}=113.01$. In-between, there are 
smaller peaks at $\mathrm{m} / \mathrm{z}=129.01$ and 145.01 . These ramp to the sulfonic acid, $\mathrm{m} / \mathrm{z}=161.003$ by jumps of 16 amu's signifying these are putative the sulfenic (or S-oxide) and sulfinic acids (the dioxide). The relative abundances of the peaks indicate that the sulfenic and sulfinic acid derivatives are relatively unstable. The rate determining step for the oxidation of $\mathrm{MMI}$ is the formation of the S-oxide.

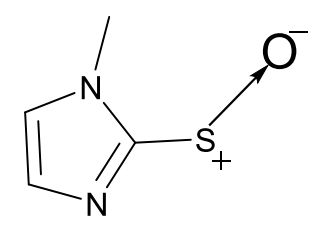

Methimazole S-oxide

Figure 3a

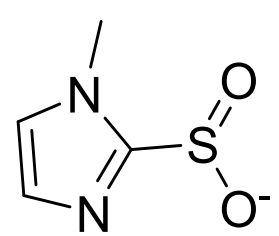

Methimazole Sulfinic Acid

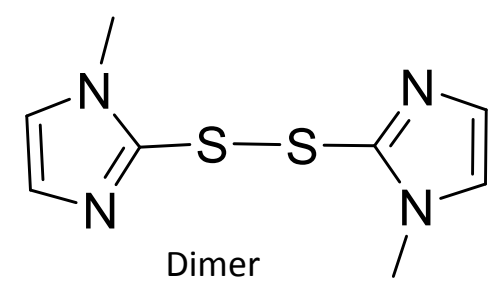

Figure 3c

Subsequent oxidation (after the first 2-electron oxidation) of the S-oxide is facile, hence the relative low abundance of these metabolites. Structures of the S-oxide and sulfinic acid metabolites are shown in Figures $3 a$ and $b$, respectively. 


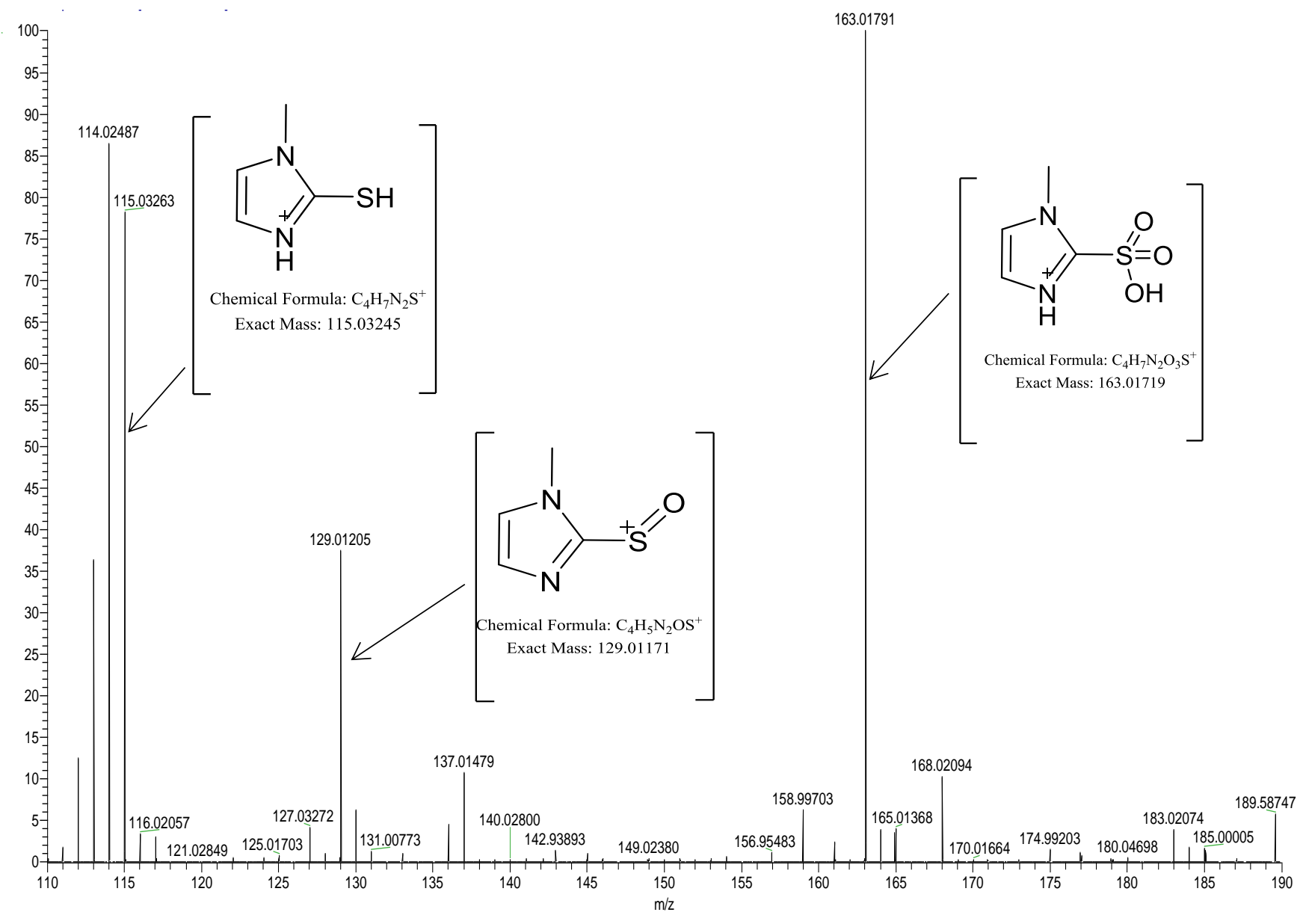

Figure 4. Positive mode. On-line ESI spectrum acquired in basic medium. This spectrum shows strong peaks for the dimer $(\mathrm{m} / \mathrm{z}=114.025$, not labeled), the sulfenic acid, and the sulfonic acid. The sulfinic acid is not observed under these conditions.

Figure 4 shows spectra derived from on-line spectra in the positive mode. As expected, the substrate now shows up at $\mathrm{m} / \mathrm{z}=115.03$. There is, surprisingly, a very strong peak for the S-oxide at 129.01 .

There is virtually no evidence for the sulfinic acid. The major metabolite, before full oxidation to the sulfonic acid, is the dimer, at $\mathrm{m} / \mathrm{z}=114.025$ (Figure $3 \mathrm{c}$ ). A slow oxidation rate will allow the electrophilic S-oxide to react with the thiol substrate in a condensation-type reaction to produce the dimer. Dimeric disulfides are not as reactive as the original thiols, and thus they accumulate. Their further oxidation rates back through the sulfenic acid are relatively slower. 


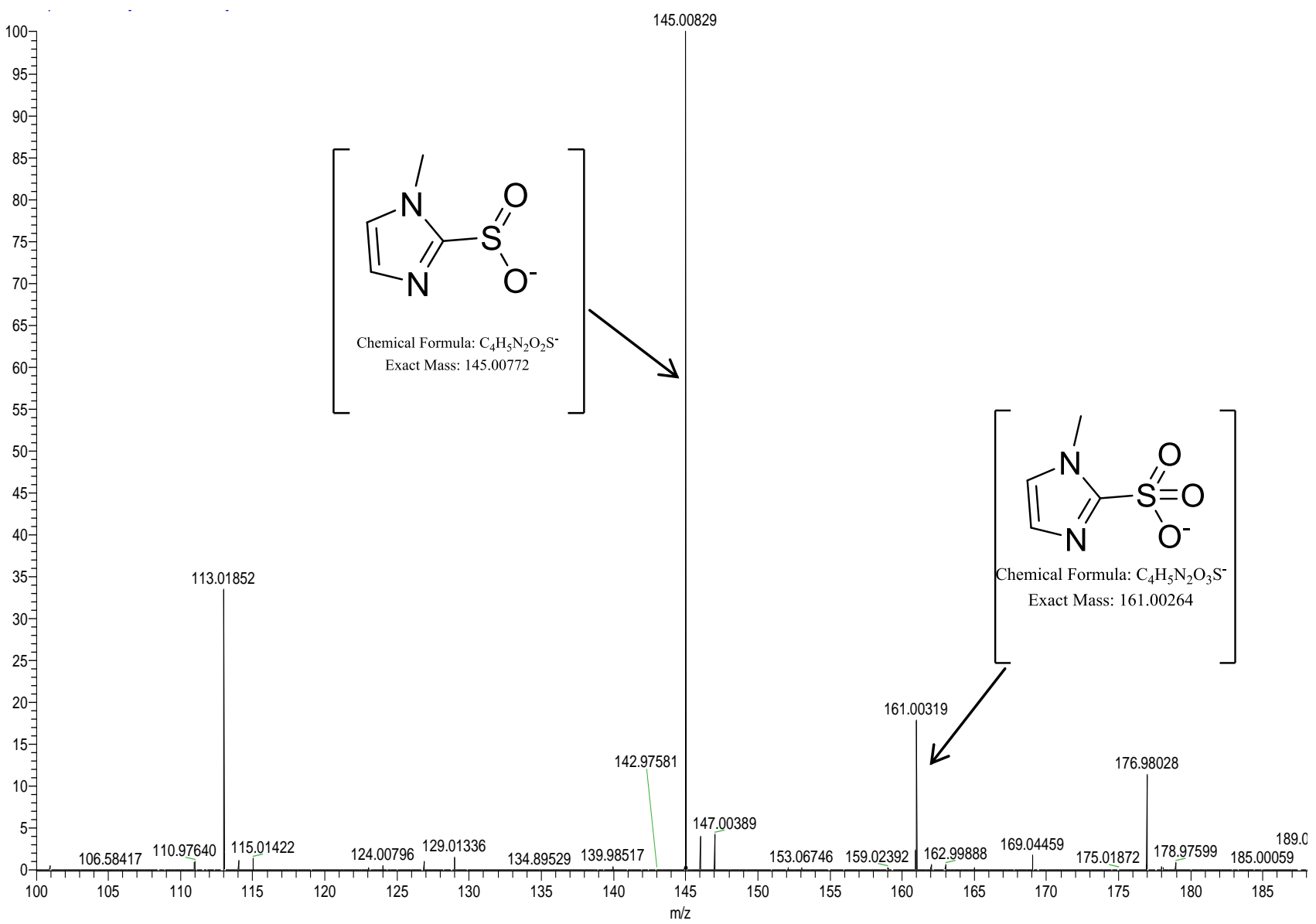

Figure 5. Negative mode. Off-line synthesis and analysis of MMI oxidation products. The products were identified with a mass accuracy of $3.93 \mathrm{ppm}$ and $3.43 \mathrm{ppm}$, absolute values, for the Methimazole sulfinic acid and Methimazole sulfonic acid respectively.

Figure 5 shows the batch off-line experimental spectra. Incubation period was long enough for the nearfull completion of the reaction. Since this is a batch environment, with no outflow of unreacted metabolites, facile reactions were able to react to completion. In contrast, negligible amounts of Soxide are observed; the major metabolite is the dioxide, methimazole sulfinic acid. Since the batch environment is constantly in an oxidizing environment, the highly labile sulfenic acid is not observed. 


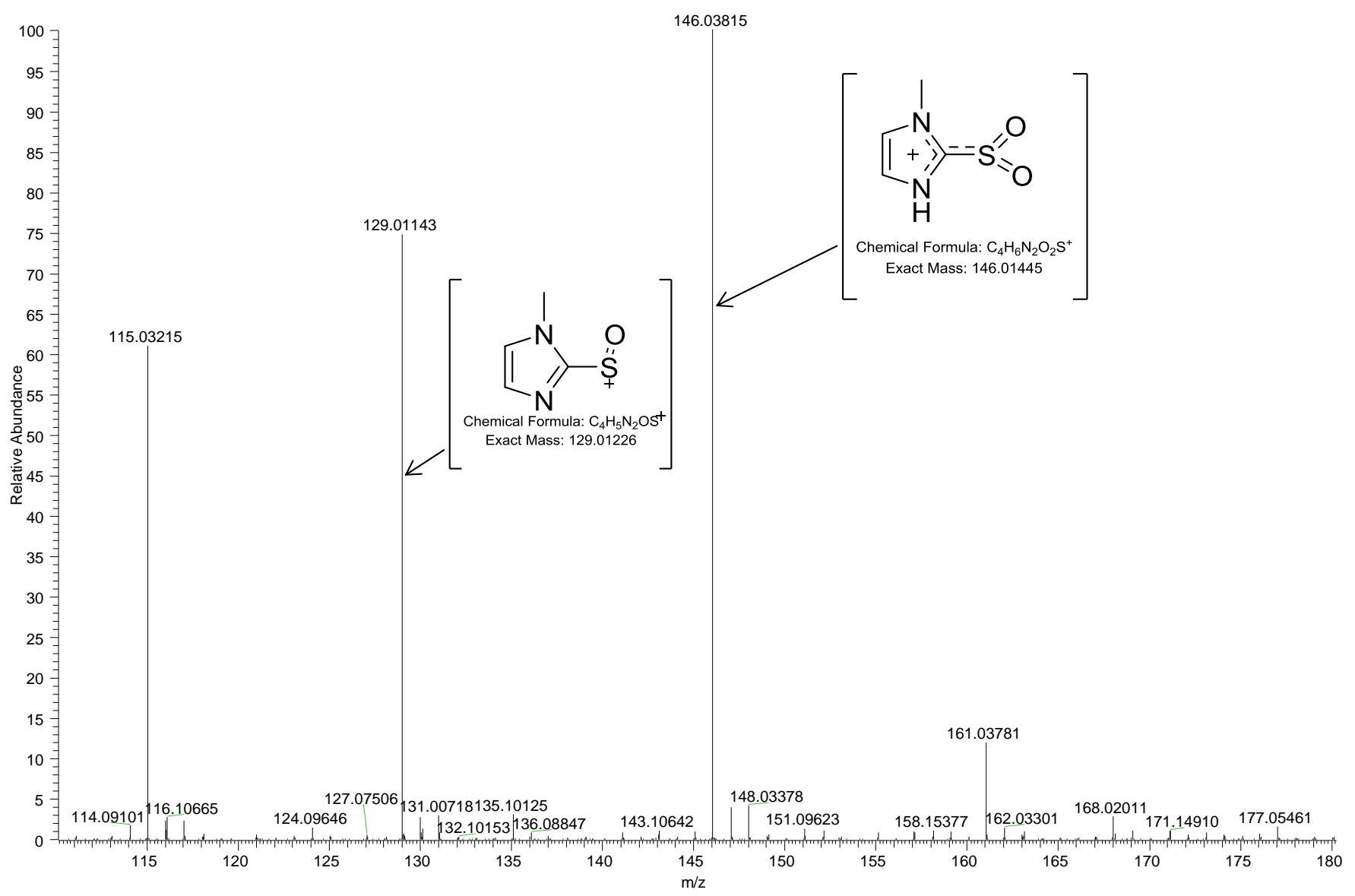

Figure 6. Off-line Positive mode. ESI spectrum showing the oxides of Methimazole. Electrochemical oxidation can result in ionization of neutral molecules that are difficult to detect, resulting in only positively charged ions that can be detected in this mode.

The positive mode results of the batch process are shown in Figure 6 . Basic environments destabilize electrophilic intermediates, and acidic environments stabilize them. Thus, in Figure 6 , the S-oxide is observed, as well as the sulfinic acid. 


\section{Electrospray lonization results in acidic media.}

The Methimazole dimer was the predominant product in acidic medium (Figure 3c, structure M4).

Figure 7 shows strong peaks for the singly charged dimer at $\mathrm{m} / \mathrm{z}=227.04$ and the doubly charged species at $\mathrm{m} / \mathrm{z}=114.03$.

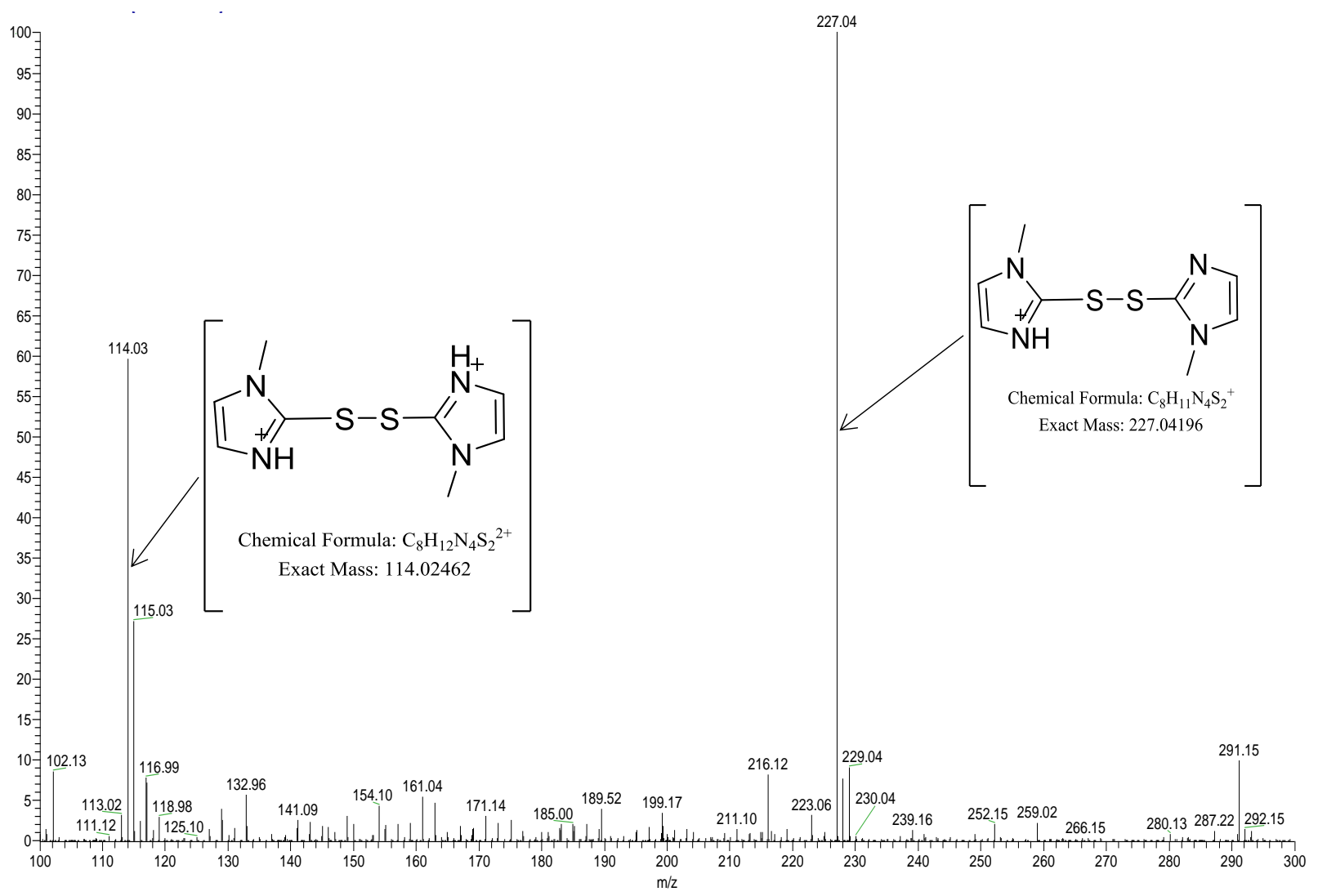

Figure 7. Positive mode ESI in acidic medium.

\section{Experimental procedure for trapping experiment}

A $100 \mu \mathrm{M}$ sample of $\mathrm{MMI}$ in $20 \%$ methanol with $80 \%$ ammonium buffer, was infused through the electrochemical cell at a flow rate of $10 \mathrm{uL} /$ minute. A solution of $\mathrm{N}$ acetyl cysteine, $100 \mu \mathrm{M}$ from another 
syringe was then infused at the same rate, the effluent from both channels combined and mixed at a Tjunction flowing into the cavity of mass spectrometer as shown in Figure 1.

\section{Experimental results derived from trapping.}

Figure 8 shows the ESI spectrum derived from the use of $\mathrm{N}$-acetylcysteine (NAC) as the trap. One strong peak is observed, of a 1:1 adduct of NAC with MMI. The experimental setup involved flowing NAC through a T-junction into the flow going into the spectrometer cavity (see Figure 1 schematic). Thus NAC was not electrochemically oxidized. It combined with the effluent from the electrochemical cell as a reducing thiol. It reacts strongly with any electrophilic species from the electrochemical cell. The absence of symmetric disulfides is important: it means no radicals are involved in this mechanism. The only electrophile present in the electrochemical oxidation cell is the S-oxide. The decrease in abundance of metabolite and formation of the conjugate strongly indicates that it is the reactive metabolite. The sulfinic and sulfenic acids are not strong electrophiles. Figure 8 thus shows that the sulfenic acid is very stable in the oxidizing environment but is quickly and quantitatively deactivated in a reducing environment such as in the presence of NAC. This (a reducing environment) is the expected medium in the physiological environment. 


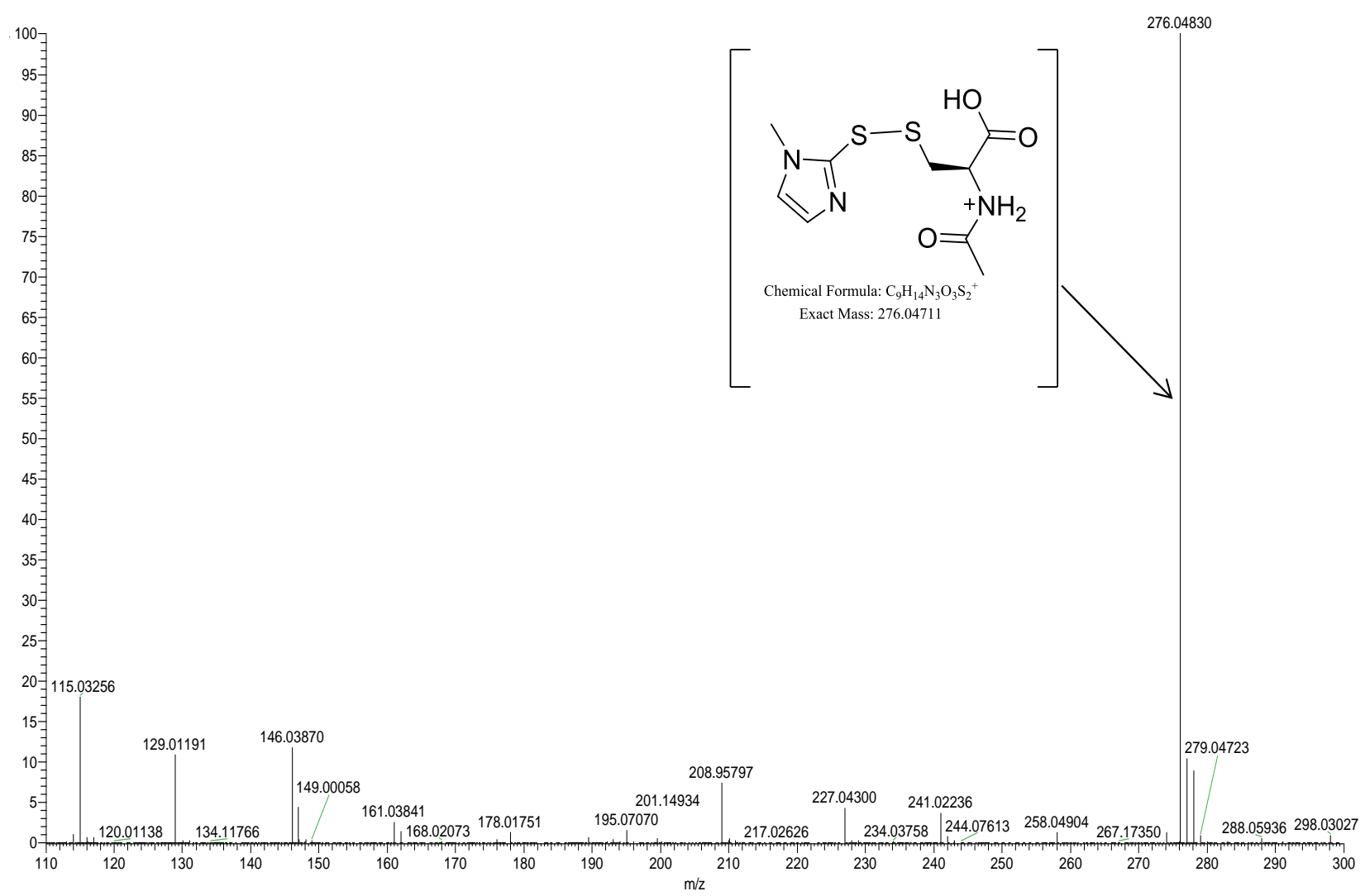

Figure $8 \mathrm{MMI}$ metabolite conjugate with $\mathrm{N}$-acetyl cysteine. There is one dominant adduct 1:1 adduct of the $\mathrm{N}$-acetylcysteine and $\mathrm{MMI}$. There is a small peak for the S-oxide and an even smaller peak for the dioxide.

\section{Mechanism.}

All the data derived from electrochemical oxidations suggests a very strong case for a viable S-oxide.

This has been, previously, an elusive metabolite. Very strong and stable peaks have been observed in Figures 4 and 6 . Figure 2, though not showing a strong sulfenic acid peak, does show a peak for the dimer. This dimer is derived from a sulfenic acid (vide supra)

The oxidation pathway has a direct correlation with the physiological effects of MMI. The standard accepted oxidation pathway of sulfur-based drugs is through S-oxygenation, though the mechanism of this S-oxygenation differs with specific compounds based on the environment of the sulfur group. All $\beta$ amino sulfur compounds are oxidized in vivo and in vitro to as far as the sulfonic acid without cleavage of the $\mathrm{C}-\mathrm{S}$ bond. Thus most biologically active molecules such as cysteine, glutathione, and cysteamine show this distinct oxidation pathway. ${ }^{28-30}$ In the presence of excess oxidant, the stoichiometry of the 
reaction was found to be strictly 1:3; with formation of the sulfonic acid with very little detectable sulfate by $\mathrm{BaSO}_{4}$ precipitation:

$$
3 \mathrm{Br}_{2}+\mathrm{RSH}+3 \mathrm{H}_{2} \mathrm{O} \rightarrow \mathrm{RSO}_{3} \mathrm{H}(\text { Structure } \mathrm{M} 3)+6 \mathrm{H}^{+}+6 \mathrm{Br}^{-}
$$

Most deleterious effects of bioactive compounds are ascribed to the reactive metabolites produced during the first pass metabolism ${ }^{31}$. By nature of the physiological environment, such reactive metabolites are never observed. The first set of experiments carried out to detect possible reactive intermediates of MMI involved its reaction with aqueous bromine. This is a 'clean' and strong oxidant that oxidizes in 2 one-electron steps to produce only $\mathrm{Br}^{-}$as its reduced product:

$$
\frac{1}{2} \mathrm{Br}_{2}(\mathrm{aq})+\mathrm{e}-\rightarrow \mathrm{Br}-\quad \mathrm{E}^{\circ} / \mathrm{V}=1.09
$$

This affords the determination of the possible metabolites without interference from the oxidant. A 1:1 mixture of $\mathrm{MMI}$ and aqueous bromine imparts a 2-electron oxidation which should limit oxidation of MMI to the sulfenic acid. If the sulfenic acid is stable enough, it should be observed in the ESI spectrum. Figure 9 shows a spectrum of a 1:1 mixture of $\mathrm{MMI}$ and aqueous bromine. Even though the spectrum shows the sulfinic and sulfonic acids; the major metabolite is surprisingly, the S-oxide (Figure 3a, Structure M1). In the absence of the reducing physiological environment, this highly unstable, electrophilic S-oxide can be stabilized. Unstable S-oxides are known to disproportionate or to form thiosulfinates. They are also expected to react with the nucleophilic remaining thiol to produce a dimer:

$$
\mathrm{RSOH}+\mathrm{RSH} \rightarrow \mathrm{RSSR}+\mathrm{H}_{2} \mathrm{O}
$$

Under insufficient oxidant equivalents, the substrate will partition into its most stable intermediates at those conditions. Spectrum in Figure 9 shows that the sulfinic acid, as expected, is not as stable as the sulfonic acid, which is the final oxidation product. This spectrum was taken after complete consumption of bromine and before full rearrangement of the sulfur compounds as evidenced by the coexistence of the S-oxide and the thiol. The spectrum obtained in Figure 9 was derived from unbuffered solutions. 
$\mathrm{pH}$ of the reaction solution decreased due to formation of $\mathrm{H}^{+}$according to the reaction stoichiometry. Thus the $\mathrm{m} / \mathrm{z}$ peak at 116.99 can be ascribed to the substrate, and the 115.03 to the dimer. After long incubation periods the substrate peak disappears completely, with a concomitant increase in the dimeric species and other unidentifiable thiosulfinates. The sulfenic and sulfinic acids still show up at the expected $\mathrm{m} / \mathrm{z}$ values because of their acidity, and thus unlikely to be further protonated despite the highly acidic product environment. 


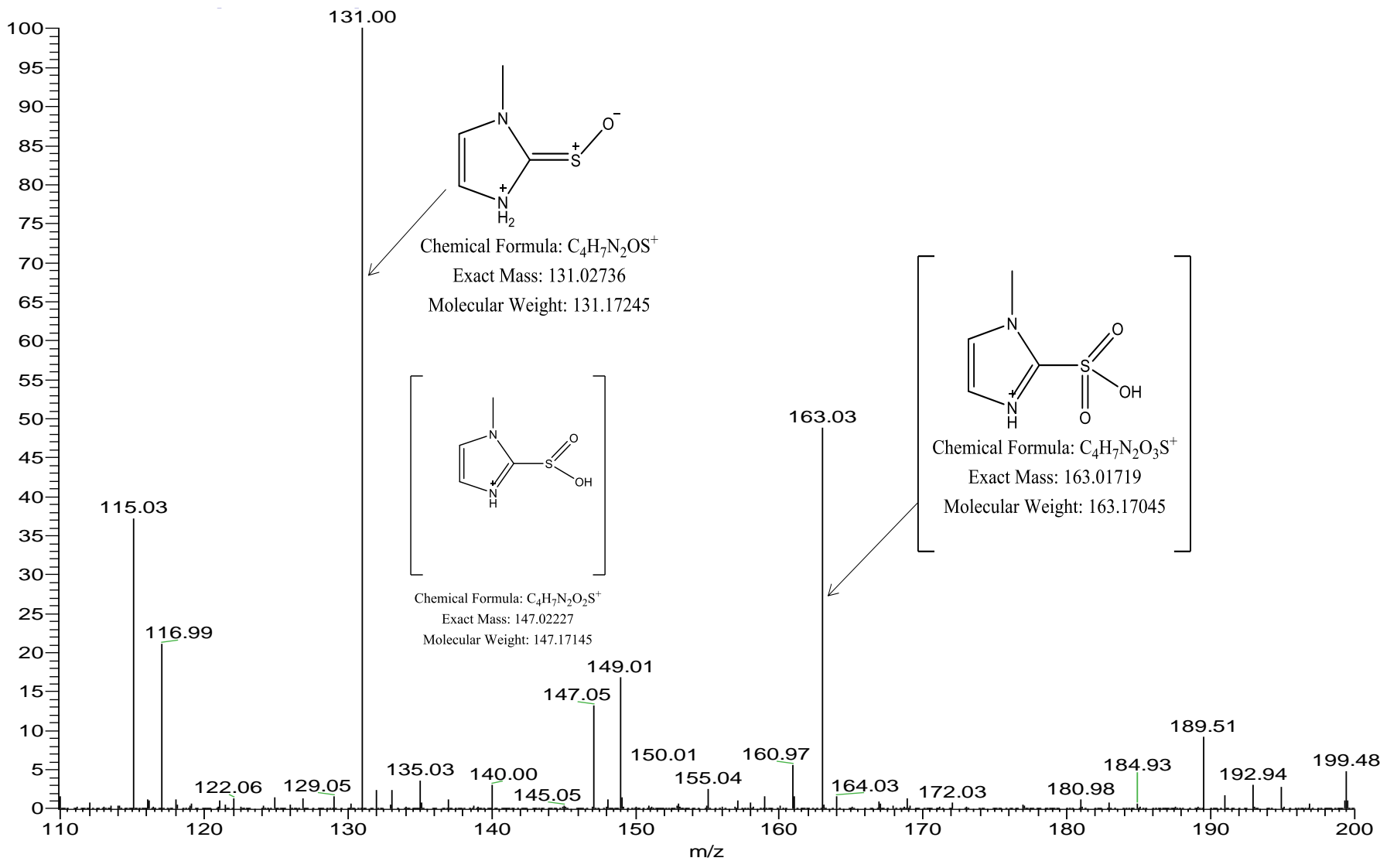

Figure 9. ESI spectrum of oxidation of methimazole using bromine in a 1:1 mixture ratio. As expected, the sulfenic acid is the major metabolite in these conditions of excess reductant.

\section{Proposed mechanism for electrochemical oxidation of methimazole}

Our experimental results can now allow us to produce a plausible mechanism of electrochemical oxidation of MMI. This is shown in Scheme 1. Electrochemical oxidation of sulfur-based drugs is complicated by the fact that sulfur centers generally oxidize via 2-electron jumps, while electrochemical abstractions generally involve a single electron transfer at a time. Starting with the thiol center would produce a sulfur-based thiyl-type radical on abstraction of the first electron. This would immediately precipitate a cascade of free radical reactions with a strong formation of the dimeric disulfide. Our results thus lead us to the conclusion that the abstraction of the first electron results in the formation of 
a positive charge delocalized mostly over the $\mathrm{C}-\mathrm{N}-\mathrm{C}$ network and not a sulfur-based radical. Zwitterionic forms of thioureido oxo-acids have similarly been described. ${ }^{32}$

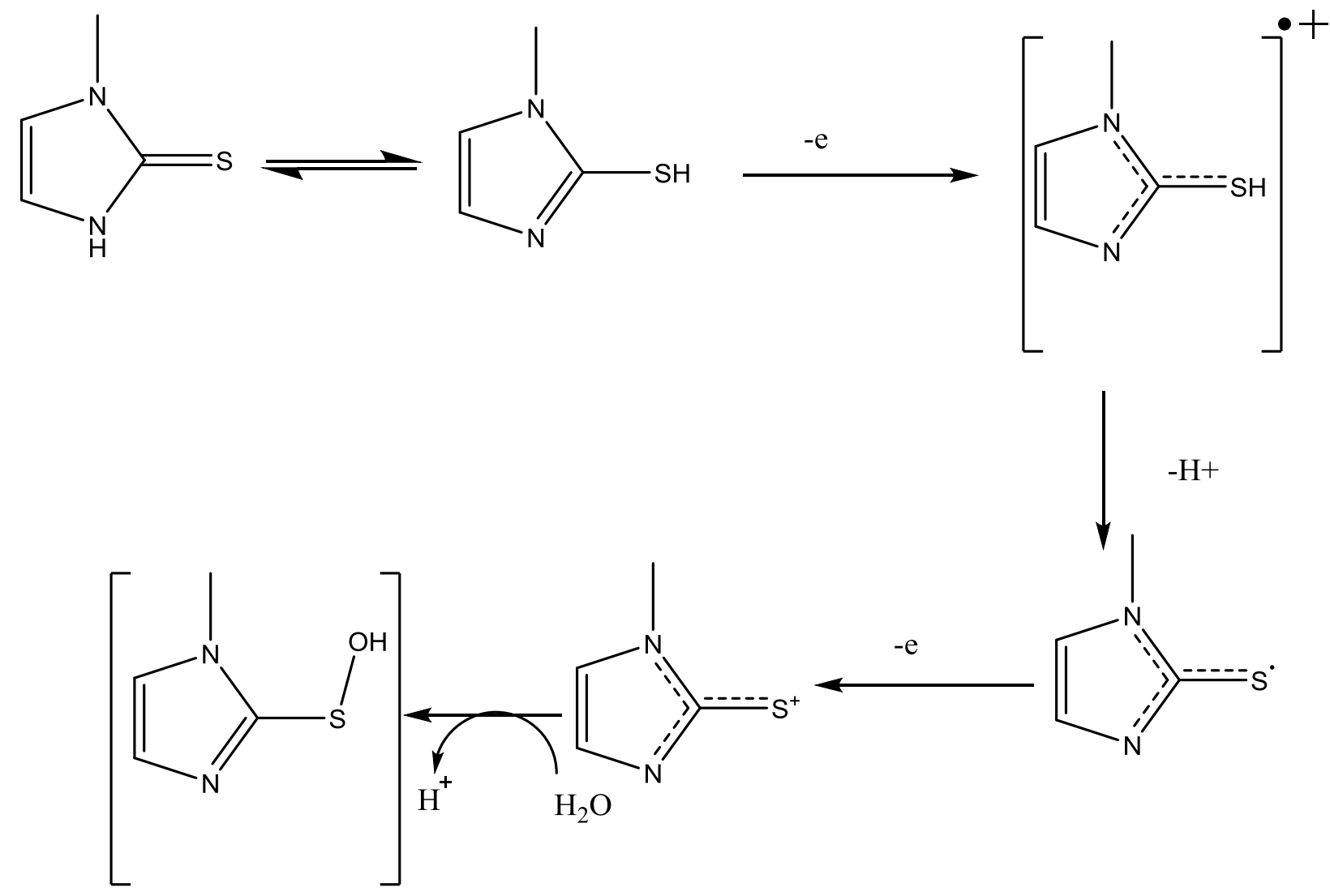

Scheme 1.

\section{Comparison with biological metabolism.}

In general, the electrochemical species generated from electrochemical oxidation of $\mathrm{MMI}$, shown here in this manuscript, are similar to metabolites obtained from in vitro incubations with microsomes.

There are other products obtained with microsomes that were not obtained here (See Table 1). The 
electrochemical process is a 'cleaner' oxidation environment than that afforded by microsomes. Our electrochemical oxidation platform involves the most easily oxidizable part of the molecule without any regard to other parameters present in bimolecular activations such as stereochemistry. Thus one would expect a compact set of metabolites, while microsomal oxidations would produce a broader spectrum of possible metabolites. The major metabolites observed in microsomal oxidations have been echoed by our electrochemical system. The maintenance of an oxidizing environment in the electrochemical environment has allowed for the observation of those highly electrophilic intermediates that would otherwise have been quenched by the reducing physiological environment. Metabolism by microsomes involves primarily the flavin-containing monooxygenases (FMO's) and the Cytochromes P450 group of enzymes. FMO's are a family of drug-metabolizing enzymes that use FAD, NADPH and molecular oxygen to catalyze the oxygenation of a large number of xenobiotics containing 'soft' nucleophiles such as sulfur, phosphorus and nitrogen..$^{33,34}$ These FMO's convert xenobiotics into polar metabolites by adding oxygen so that they can subsequently be easily eluted through the kidneys. ${ }^{35}$ While initially thought to be a single enzyme; FMO's are now known to contain at least 11 isoforms, with 5 of them having been characterized to date. ${ }^{36,37}$ Since $\mathrm{MMI}$ is a well-tolerated drug for hyperthyroidism, its in vitro and in vivo metabolism has been extensively studied. Human, rat and pig microsomal incubations with MMI have concluded that the major activation of $\mathrm{MMI}$ is performed by $\mathrm{FMO}^{\prime}$ 's rather than CYP450's. Presence of FMO's however, seemed to deactivate CYP-based bioactivations, and it has since been established that MMI is a competitive inhibitor of FMO's. ${ }^{38,39}$ Isolation of the effect of CYP450's can be determined by heating, which deactivates FMO's or addition of N-octylamine, which inhibits FMO bioactivations. The variety of metabolites obtained which involved ring cleavage to obtain methyl thiourea and its oxo-acids (structures M11-13 in Table 1) can be attributed to CYP450-mediated bioactivations. This can be justified through the initial epoxidation of MMI (structure M9) whose subsequent hydrolysis product of a 1,2 dihydroxy moiety can easily cleave the $\mathrm{C}-\mathrm{C}$ bond by even the mildest oxidants. Thus one expects a 
wide variety of possible metabolism products when microsomal oxidations are utilized to metabolize MMI. The major pathway of microsomal bioactivation of MMI involves S-oxygenation, with the sulfinic acid (structure M2) as the major metabolite. While the sulfenic acid has been postulated (structure M1), microsomal incubations had never been able to isolate it; even though formation of $M 2$ has to pass through M1. Our study has been able to isolate this formerly postulated metabolite. The deactivation of CYP450's is clearly related to formation of a highly reactive electrophilic metabolite of MMI derived from FMO's. This has been observed with the oxidation of a novel kinase inhibitor, TG100435. ${ }^{40,41}$ Its bioactivation by FMO's gives an S-oxygenated S-Oxide, TG100855, which not only deactivates Cyp450's but is also reduced back to TG100435 during the deactivation. Thus the products, kinetics and dynamics of microsomal bioactivations are expected to be complex. Our electrochemical oxidations simplify this process. This makes evaluation of physiological effects, especially toxicities, easier to determine.

\section{Conclusion}

Bioactivations of sulfur-based drugs have always been difficult to determine. Sulfur chemistry is generally complex, characterized by free radical mechanisms and formation of polymeric species. Toxicities of sulfur-based drugs have been attributed to formation of highly reactive sulfur oxo-acids and depletion of glutathione by the formation of reactive metabolites. The most-postulated metabolite, the sulfenic acid, had never been isolated until now, using electrochemistry on-line with electrospray ionization.

\section{Acknowledgements.}

This work was supported by research grant number CHE-1056366 from the National Science Foundation and a partial Research Professor allocation from the University of KwaZulu-Natal, Durban, South Africa. 
1. Zhanga, D.; Luo, G.; Dingc, X.; Lud, G.; Lu.C Preclinical experimental models of drug metabolism and disposition in drug discovery and development. Acta Pharmaceutica Sinica B 2012, 2 (6), 549-561.

2. Arora, T.; Mehta, A. K.; Joshi, V.; Mehta, K. D.; Rathor, N.; Mediratta, P. K.; Sharma, K. K. Substitute of Animals in Drug Research: An Approach Towards Fulfillment of 4R's. Indian Journal of Pharmaceutical Sciences 2011, 73 (1), 1-6.

3. Lohmann, W.; Karst, U. Biomimetic modeling of oxidative drug metabolism. Analytical and Bioanalytical Chemistry 2008, 391 (1), 79-96.

4. Lohmann, W.; Karst, U. Generation and identification of reactive metabolites by electrochemistry and immobilized enzymes coupled on-line to liquid chromatography/mass spectrometry. Analytical Chemistry 2007, 79 (17), 6831-6839.

5. Baumann, A.; Lohmann, W.; Schubert, B.; Oberacher, H.; Karst, U. Metabolic studies of tetrazepam based on electrochemical simulation in comparison to in vivo and in vitro methods. Journal of Chromatography A 2009, 1216 (15), 3192-3198.

6. Gamache, P.; Smith, R.; McCarthy, R.; Waraska, J.; Acworth, I.; Williams, D. A. Redox profiling of pharmaceuticals using on-line electrochemistry-mass spectrometry. Drug Metabolism Reviews 2003, 35, 84.

7. Kalgutkar, A. S.; Gardner, I.; Obach, R. S.; Shaffer, C. L.; Callegari, E.; Henne, K. R.; Mutlib, A. E.; Dalvie, D. K.; Lee, J. S.; Nakai, Y.; O'Donnell, J. P.; Boer, J.; Harriman, S. P. A comprehensive listing of bioactivation pathways of organic functional groups. Curr. Drug Metab 2005, 6 (3), 161-225.

8. Jurva, U.; Wikstrom, H. V.; Weidolf, L.; Bruins, A. P. Comparison between electrochemistry/mass spectrometry and cytochrome $\mathrm{P} 450$ catalyzed oxidation reactions. Rapid Communications in Mass Spectrometry 2003, 17 (8), 800-810.

9. Chipiso, K.; Mbiya, W.; Morakinyo, M. K.; Simoyi, R. H. Oxyhalogen-Sulfur Chemistry: Kinetics and Mechanism of Oxidation of N-Acetyl-I-methionine by Aqueous lodine and Acidified lodate. Australian Journal of Chemistry 2014, 67 (4), 626-635.

10. Chigwada, T.; Mbiya, W.; Chipiso, K.; Simoyi, R. H. S-oxygenation of thiocarbamides V: oxidation of tetramethylthiourea by chlorite in slightly acidic media. J. Phys. Chem. A 2014, 118 (31), 5903-5914.

11. Zuniga, F. I.; Loi, D.; Ling, K. H. J.; Tang-Liu, D. D. S. Idiosyncratic reactions and metabolism of sulfur-containing drugs. Expert Opinion on Drug Metabolism \& Toxicology 2012, 8 (4), 467-485. 
12. Israeli, A.; Or, R.; Leitersdorf, E. Captopril-associated transient aplastic anemia. Acta Haematol. 1985, 73 (2), 106-107.

13. Jaffe, I. A. Adverse effects profile of sulfhydryl compounds in man. Am. J. Med. 1986, 80 (3), 471476.

14. Kim, C. R.; Maley, M. B.; Mohler, E. R., Jr. Captopril and aplastic anemia. Ann. Intern. Med. 1989, $111(2), 187-188$.

15. Otsuka, F.; Noh, J. Y.; Chino, T.; Shimizu, T.; Mukasa, K.; Ito, K.; Ito, K.; Taniyama, M. Hepatotoxicity and cutaneous reactions after antithyroid drug administration. Clinical Endocrinology 2012, 77 (2), 310-315.

16. Rivkees, S. A.; Szarfman, A. Dissimilar Hepatotoxicity Profiles of Propylthiouracil and Methimazole in Children. Journal of Clinical Endocrinology \& Metabolism 2010, 95 (7), 3260-3267.

17. Uetrecht, J. P. Reactive metabolites and idiosyncratic toxicity. Drug Metabolism Reviews 2006, 38, 35-36.

18. Uetrecht, J. Idiosyncratic drug reactions: Past, present, and future. Chemical Research in Toxicology 2008, 21 (1), 84-92.

19. Jurva, U.; Holmen, A.; Gronberg, G.; Masimirembwa, C.; Weidolf, L. Electrochemical generation of electrophilic drug metabolites: Characterization of amodiaquine quinoneimine and cysteinyl conjugates by MS, IR, and NMR. Chemical Research in Toxicology 2008, 21 (4), 928-935.

20. Johansson, T.; Jurva, U.; Gronberg, G.; Weidolf, L.; Masimirembwa, C. Novel Metabolites of Amodiaquine Formed by CYP1A1 and CYP1B1: Structure Elucidation Using Electrochemistry, Mass Spectrometry, and NMR. Drug Metabolism and Disposition 2009, 37 (3), 571-579.

21. Madsen, K. G.; Skonberg, C.; Jurva, U.; Cornett, C.; Hansen, S. H.; Johansen, T. N.; Olsen, J. Bioactivation of diclofenac in vitro and in vivo: Correlation to electrochemical studies. Chemical Research in Toxicology 2008, 21 (5), 1107-1119.

22. Mizutani, T.; Yoshida, K.; Murakami, M.; Shirai, M.; Kawazoe, S. Evidence for the involvement of $\mathrm{N}$-methylthiourea, a ring cleavage metabolite, in the hepatotoxicity of methimazole in glutathione-depleted mice: Structure-toxicity and metabolic studies. Chemical Research in Toxicology 2000, 13 (3), 170-176.

23. Kedderis, G. L.; Rickert, D. E. Loss of Rat-Liver Microsomal Cytochrome-P-450 During Methimazole Metabolism - Role of Flavin-Containing Monooxygenase. Drug Metabolism and Disposition 1985, 13 (1), 58-61.

24. Aslanoglu, M.; Peker, N. Potentiometric and voltammetric determination of methimazole. Journal of Pharmaceutical and Biomedical Analysis 2003, 33 (5), 1143-1147. 
25. Sun, J. Y.; Zheng, C. Y.; Xiao, X. L.; Niu, L.; You, T. Y.; Wang, E. K. Electrochemical detection of methimazole by capillary electrophoresis at a carbon fiber microdisk electrode.

Electroanalysis 2005, 17 (18), 1675-1680.

26. Wang, Y. Z. Electrochemical determination of methimazole based on the acetylene black/chitosan film electrode and its application to rat serum samples.

Bioelectrochemistry 2011, 81 (2), 86-90.

27. ESA Inc Coulochem III (50W) Reference Manual: Chapter 4. 2004; pp 5-7.

28. Darkwa, J.; Mundoma, C.; Simoyi, R. H. Antioxidant chemistry - Reactivity and oxidation of DLcysteine by some common oxidants. Journal of the Chemical Society-Faraday Transactions 1998, 94 (14), 1971-1978.

29. Darkwa, J.; Olojo, R.; Chikwana, E.; Simoyi, R. H. Antioxidant chemistry: Oxidation of L-cysteine and its metabolites by chlorite and chlorine dioxide. Journal of Physical Chemistry $A$ 2004, 108 (26), 5576-5587.

30. Darkwa, J.; Olojo, R.; Olagunju, O.; Otoikhian, A.; Simoyi, R. Oxyhalogen-sulfur chemistry: Oxidation of $\mathrm{N}$-acetylcysteine by chlorite and acidic bromate. Journal of Physical Chemistry A 2003, 107 (46), 9834-9845.

31. Attia, S. M. Deleterious effects of reactive metabolites. Oxidative Medicine and Cellular Longevity 2010, 3 (4), 238-253.

32. Simoyi, R. H.; Svarovsky, S. A.; Mundoma, C. M.; Makarov, S. V. Reactive oxygen species and sulfoxyl radicals in the aerobic decomposition of thioureas dioxides. Abstracts of Papers of the American Chemical Society 1999, 218, U375.

33. Henderson, M. C.; Krueger, S. K.; Stevens, J. F.; Williams, D. E. Human flavin-containing monooxygenase form 2 S-oxygenation: Sulfenic acid formation from thioureas and oxidation of glutathione. Chemical Research in Toxicology 2004, 17 (5), 633-640.

34. Krueger, S. K.; Williams, D. E. Mammalian flavin-containing monooxygenases:

structure/function, genetic polymorphisms and role in drug metabolism. Pharmacol. Ther. 2005, 106 (3), 357-387.

35. Eswaramoorthy, S.; Bonanno, J. B.; Burley, S. K.; Swaminathan, S. Mechanism of action of a flavin-containing monooxygenase. Proc. Natl. Acad. Sci. U. S. A 2006, 103 (26), 98329837.

36. Cashman, J. R.; Zhang, J. Human flavin-containing monooxygenases. Annu. Rev. Pharmacol. Toxicol. 2006, 46, 65-100.

37. Koukouritaki, S. B.; Hines, R. N. Flavin-containing monooxygenase genetic polymorphism: impact on chemical metabolism and drug development. Pharmacogenomics. 2005, 6 (8), 807822. 
38. Hsu, D. Z.; Chu, P. Y.; Li, Y. H.; Chandrasekaran, V. R.; Liu, M. Y. Role of flavin-containingmonooxygenase-dependent neutrophil activation in thioacetamide-induced hepatic inflammation in rats. Toxicology 2012, 298 (1-3), 52-58.

39. Tian, X.; Sun, X.; Su, J. Biochemical mechanisms for metaflumizone resistance in beet armyworm, Spodoptera exigua. Pestic. Biochem. Physiol 2014, 113, 8-14.

40. Hu, S. X.; Soll, R.; Yee, S.; Lohse, D. L.; Kousba, A.; Zeng, B.; Yu, X.; McPherson, A.; Renick, J.; Cao, J.; Tabak, A.; Hood, J.; Doukas, J.; Noronha, G.; Martin, M. Metabolism and pharmacokinetics of a novel Src kinase inhibitor TG100435 ([7-(2,6-dichloro-phenyl)-5methyl-benzo[1,2,4]triazin-3-yl]-[4-(2-pyrrolidin-1-y l-ethoxy)-phenyl]-amine) and its active $\mathrm{N}$-oxide metabolite TG100855 ([7-(2,6-dichloro-phenyl)-5methylbenzo[1,2,4]triazin-3-yl]-\{4-[2-(1-oxy-pyrrolid in-1-yl)-ethoxy]-phenyl\}-amine). Drug Metab Dispos. 2007, 35 (6), 929-936.

41. Kousba, A.; Soll, R.; Yee, S.; Martin, M. Cyclic conversion of the novel Src kinase inhibitor [7-(2,6dichloro-phenyl)-5-methyl-benzo[1,2,4]triazin-3-yl]-[4-(2-pyrrolidin-1-yl -ethoxy)phenyl]-amine (TG100435) and Its $\mathrm{N}$-oxide metabolite by flavin-containing monoxygenases and cytochrome P450 reductase. Drug Metab Dispos. 2007, 35 (12), 2242-2251. 